\section{A nurse-led ocular oncology clinic in Liverpool: results of a 6-month trial}

\begin{abstract}
Purpose To describe the design and implementation of a nurse-led clinic in a tertiary adult ocular oncology service and to assess its feasibility and patient satisfaction. Methods Patients with a melanocytic uveal tumour attending for review during an initial 6-month trial period were assessed in a dedicated ocular oncology clinic by an ophthalmic nurse practitioner. These were:

(1) patients who would have been discharged back to the referring hospital but whose ophthalmologist refused to continue their follow-up; (2) patients who preferred to be reviewed in our clinic; and (3) patients with a risk of metastatic disease that was increased but not enough for them to be referred to our medical oncologist. Quality assurance mechanisms were established to ensure safe practice. Patient satisfaction was assessed by means of anonymised questionnaires.

Results A total of 65 patients were seen between 1 November 2011 and 31 May 2011. The mean age was 58 years (range 16-82 years). Most lesions seen were choroidal suspicious naevi (54\%) and treated choroidal malignant melanomas (20\%). Nine (14\%) patients with an increased risk of metastatic disease attended the clinic. Nine patients (14\%) were referred back to the ophthalmologist's ocular oncology clinic, because of tumour growth in two patients, macular oedema in one, cataract in five, and conjunctival melanosis at the plaque site in one. Questionnaires showed high levels of satisfaction with the service.

Conclusion A nurse-led adult ocular oncology clinic is feasible, thanks to developments in ocular photography. It is well accepted by patients.

Eye (2012) 26, 937-943; doi:10.1038/eye.2012.62; published online 20 April 2012
\end{abstract}

Keywords: uveal tumour; follow-up; nurse practitioner
T Sandinha, G Hebbar, N Kenawy, L Hope-Stone and B Damato

\section{Introduction}

Many patients with a melanocytic uveal tumour require life-long follow-up. Such surveillance is needed to differentiate a large, benign naevus from a small, malignant melanoma and to detect local tumour recurrence of a melanoma after radiotherapy, phototherapy, or local resection. Such long-term monitoring requires examination every 6 months or annually, comparing the ophthalmoscopic or slit-lamp findings with baseline colour photographs and, in selected cases, ultrasonography. At the Liverpool Ocular Oncology Centre (LOOC), we review patients until the likelihood of tumour growth or re-growth is considered to be $\leq 1 \%$, when they are discharged back to the referring hospital. This is so that we can safely manage large numbers of high-risk patients requiring specialist care. Recent improvements in ocular photography have made it easier to detect tumour change over time and this has encouraged us to set up an ocular oncology clinic staffed by Ophthalmic Nurse

Practitioners. These nurses are highly-trained clinicians who have been authorised by their employer to work autonomously. We therefore established a nurse oncology clinic (NOC) for long-term surveillance of selected patients with an ocular tumour.

The aim of this study was to evaluate our NOC in terms of feasibility and patient satisfaction.

Materials and methods

\section{Patients}

Attendees of the NOC included: (1) patients who would have been discharged back to the referring hospital but whose ophthalmologist refused to continue their follow-up; (2) patients who preferred to be reviewed at the LOOC; and (3) patients with an increased risk of metastatic
Liverpool Ocular Oncology Centre, St Paul's Eye Unit, Liverpool, UK

Correspondence:

B Damato, University Eye Hospital, Oncology Service, Prescot Street, Liverpool L7 8XP, UK Tel: + 44 (0)151 706 3973; Fax: + 44 (0)151 7065436 . E-mail: Bertil@Damato.co.uk

Received: 22 June 2011 Accepted in revised form: 1 March 2012 Published online: 20 April 2012 
disease but who did not meet our medical oncologist's referral criteria because their 5-year survival probability exceeded $50 \%$. The patients seen at the NOC had been diagnosed with (a) a choroidal or iris naevus requiring monitoring because of atypical appearances or (b) a choroidal or iris melanoma after radiotherapy, phototherapy, or local resection. They were excluded if the tumour could not be photographed in its entirety or if baseline colour photographs were inadequate.

\section{Nurses}

The lead nurse delivering these clinics had many years of experience in ophthalmology, including vitreoretinal surgery. She had also practised as a specialist nurse in ocular oncology for 2 years, during which she had participated in outpatient clinics, assisted in theatre, cared for inpatients, and contributed to the administration of LOOC. She had received training in ocular slit-lamp examination, ophthalmoscopy, ultrasonography, and interpretation of ocular photographs. She was also supervised during her tenure in the actual nurse-led clinics by an ocular oncology fellow for 4 months. This nurse also received training from a trainee health psychologist. The nurse practitioner was free to exercise discretion when planning patient care. She was accompanied in the NOCs by other specialist ophthalmic nurses, all of whom had similar, extensive experience in ocular oncology. This was done to ensure continuity.

\section{Clinic}

The NOC was held every Thursday afternoon, after an initial period when it ran every alternate week. Each clinic had on average six patients and a maximum of ten, with all patients having previously seen a consultant at LOOC. The clinic structure and protocols were designed in conjunction with the Clinical Lead in Ocular Oncology (BED). The management protocol followed is summarised in Figure 1.

Quality checks were provided by alternating patients between the NOC and the ophthalmologist's ocular oncology clinic. The patients were reviewed every 6 months for 2 years and, if stable, annually thereafter. However, for anxious patients requiring more time, the interval between visits was reduced. These follow-up arrangements were made after discussion with the patients so that they felt involved in the decision-making process. Additional appointments were made as necessary. Patients were encouraged to telephone the nurse at any time.

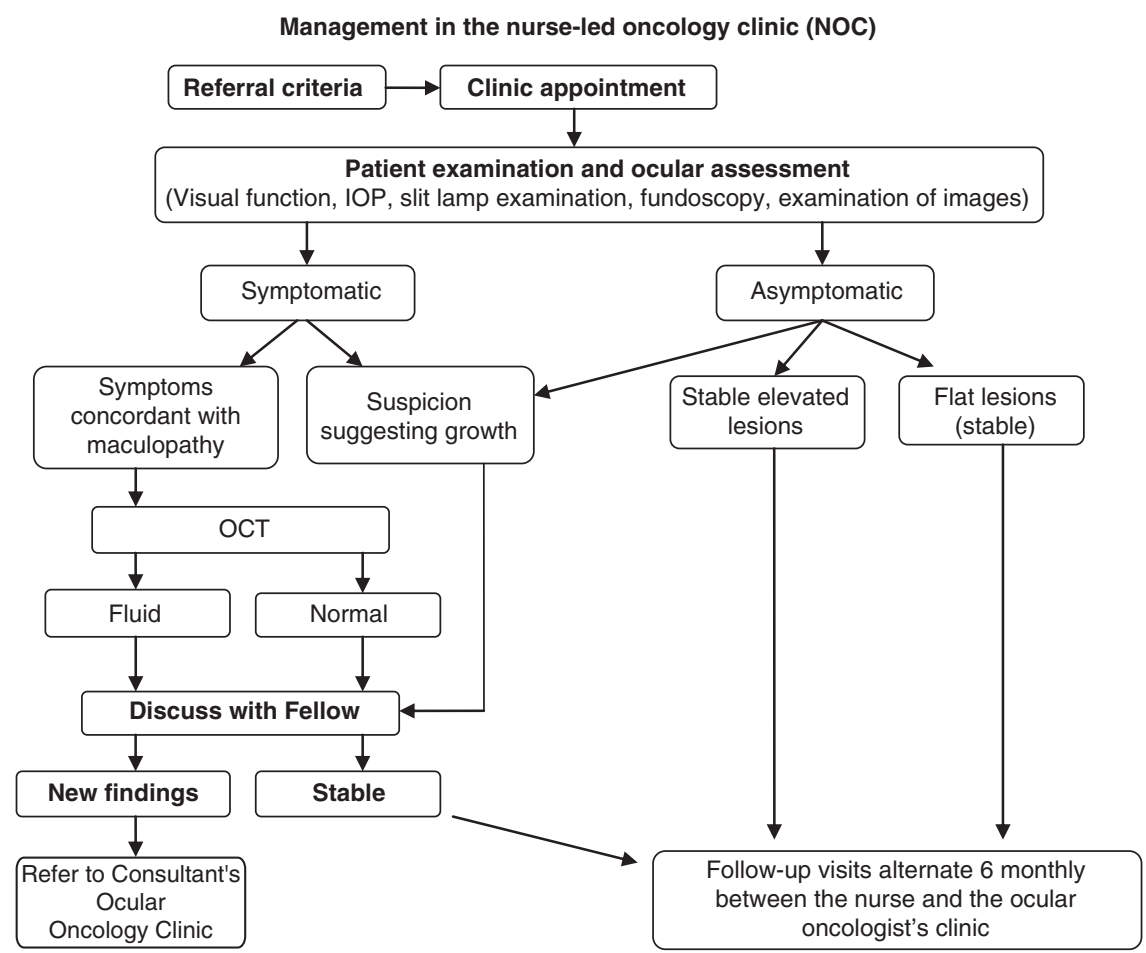

Figure 1 Diagrammatic representation of patient management in the nurse-led clinic. IOP, intraocular pressure; OCT, optical coherence tomography. 


\section{Equipment and materials}

Equipment in the clinic included: (1) a desktop computer, linked by a network to relevant imaging systems, which comprised the Optomap A10022 (Optos Ltd, Dundee, UK) (Figure 2); (2) an Eyecubed ultrasound machine ( $\mathrm{I}^{3}$ System; Ellex Ltd, Adelaide, SA, Australia); and (3) a digital voice recorder for preparing a CD-ROM recording of selected conversations, to be given to the patient. Forms included: (1) clinical data form and (2) an anonymised satisfaction questionnaire (Figure 3), which was given to each patient at the end of the consultation. Data were documented on proformas, which were filed in the casenotes and were also computerised by a fulltime data manager.

\section{Ocular assessment}

Assessment included: full ocular and systemic history, with questioning about psychological status, logMAR (logarithm of the minimal angle of resolution) visual acuity, intraocular pressure measured by applanation tonometry, slit-lamp examination, and ophthalmoscopy performed with a 90-D and/or a 20-D lens. Current photographs were compared with previous colour images, using landmarks such as retinal blood vessels and optic disc as reference points. The photographs were taken by a full-time ocular ophthalmic photographer. In selected cases, measurement of tumour thickness was performed with B-scan ultrasonography, which was performed by the nurse. If macular oedema was suspected, optical coherence tomography was undertaken and this was performed by an ophthalmic photographer.

\section{Systemic assessment}

Patients with a high risk of metastasis (ie, $>50 \%$ at 5 years) were referred to our medical oncologist, who

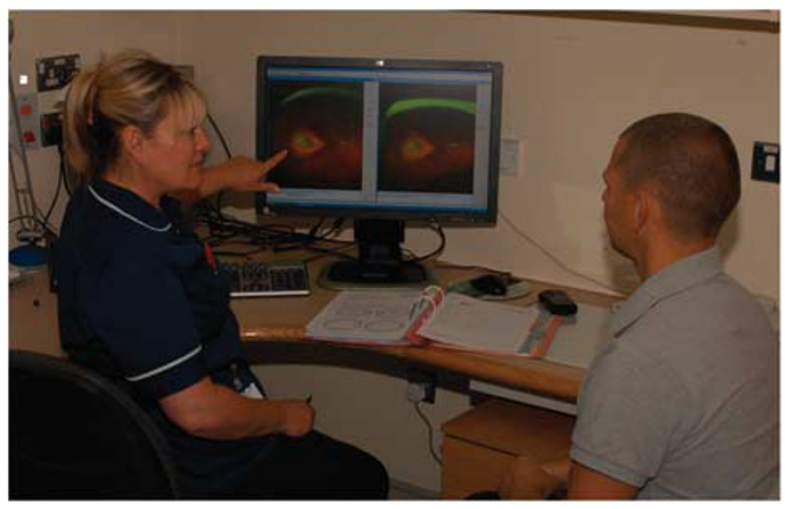

Figure 2 The nurse practitioner showing a patient his own photographs, both current and previous, using wide-angle fundus photographs, obtained with the Optos camera. entered them into a clinical trial on screening for metastasis. Patients with a low risk (ie, $<10 \%$ at 10 years) were referred to their local hospital for further care with a letter advising their ophthalmologist and general practitioner on further management. Patients with an intermediate risk were brought back to our clinic to see the consultant ocular oncologist, who discussed the prognosis according to the genetic, histological, and clinical risk factors. The consultant then agreed a personalised management plan with each patient. Patients who wished to be screened and who accepted an offer of screening in Liverpool were offered the opportunity of having an MRI scan and a blood test every

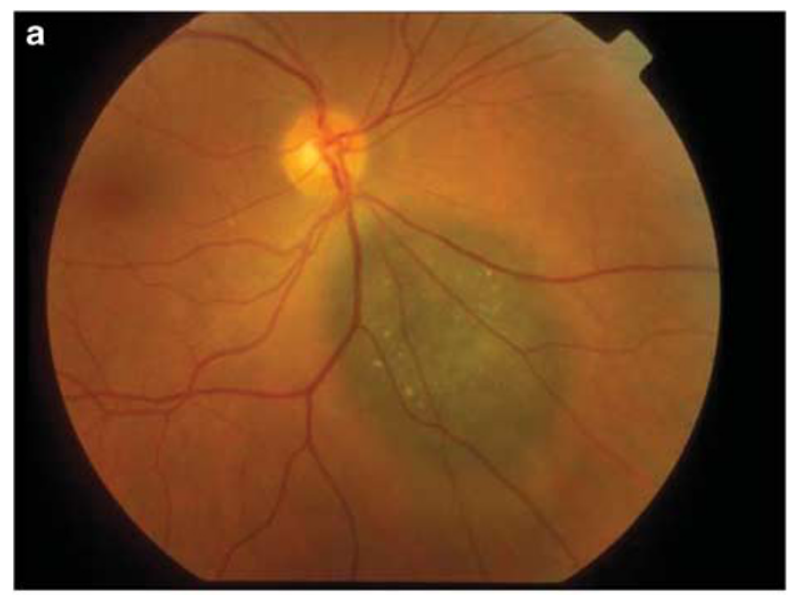

b

Figure 3 A 60-year-old woman was referred with a suspicious pigmented choroidal naevus in the right eye in 2006 (a). The lesion had drusen but no serous detachment and no visible orange pigment. On ultrasonography, the tumour had a basal diameter of $6.6 \mathrm{~mm}$ and a thickness of $1.6 \mathrm{~mm}$. The patient was discharged to a general ophthalmic clinic at our hospital for life-long monitoring. In 2011, the patient was selected at random for further surveillance at our NOC (b). The tumour was found by the nurse to have grown, with the development of clumps of orange pigment. These findings were confirmed by the consultant ocular oncologist. 
6 months, on a Thursday morning, with the result of the scan being given to the patient by the nurse on the same day. The nurse also collected blood samples for research, completed a quality of life form with the patient, and discussed any issues. If necessary, the nurse arranged for the patient to be seen by the trainee health psychologist (at 30 minutes' notice). If the scan suggested metastasis (a rare event in these patients), the nurse arranged for an ocular oncologist to speak to the patient to break the bad news and then spent time with the patient afterwards, providing counselling and support. At the end of the consultation, patients were: (a) referred back to the ocular oncology clinic; (b) referred on to a general ophthalmology clinic; or (c) reviewed at the NOC.

\section{Evaluation of the patients after enucleation}

After enucleation, patients were not reviewed at our clinic unless they required screening for metastatic disease and/or psychological support. Any oculoplastic problems were managed by their local ophthalmologist.

\section{Quality assurance}

Quality assurance mechanisms were established to ensure safe practice and to detect any suboptimal management. Competencies were assessed by reviewing audit/patient records, observing nursing practice, and oral questioning of the nurses to check knowledge and understanding. The supervising doctor signed a form when the competencies had been achieved (Table 1).

\section{Psychological assessment}

For several years, all patients treated for uveal melanoma at LOOC have been invited to complete quality of life questionnaires 6 months after their initial ocular treatment and annually thereafter. These patient-related outcome measures (PROMs) were assessed so as to be able to audit and improve patient care at our centre, as well as enhancing counselling and decision-making. These PROMs measured: (1) ophthalmological symptoms, using the EORTC OPT $30 ;^{1}$ (2) quality of life, with the Functional Assessment of Chronic Illness Therapy; ${ }^{2}$ (3) mood with the Hospital Anxiety and Depression Scale (HADS); ${ }^{3}$ and (4) social support. ${ }^{4}$ The questionnaire documented basic demographic details, major health problems, and life events. For patients attending the clinic and requiring assessment by a trainee health psychologist, the assessment was guided by the Stress and coping model, ${ }^{5}$ which identifies the patients' main challenges and concerns, how they cope with them, and what support they have. If it was judged clinically appropriate, patients were screened for mood disturbances using the HADS and emotional distress using the distress thermometer. ${ }^{6}$ These patients were also asked to complete a patient satisfaction questionnaire, constructed specifically to evaluate the quality of the NOC. This questionnaire, which was anonymised, was given to the patients after every visit.

\section{Results}

Between November 2010 and May 2011, 65 patients attended the nurse-led clinic (Table 2). These patients (28 male and 37 female) had a mean age of 58 years (range 16-82 years).

\section{Untreated patients}

A total of 35 patients were observed for an untreated melanocytic tumour of indeterminate malignancy

Table 1 Competencies required from the nurse practitioner

\begin{tabular}{l}
\hline Competency assessment \\
\hline Assesses patient's condition, taking into account general health, psychological state, and social needs. \\
Records history accurately. \\
Checks visual acuity accurately. \\
Examines patients using the slit lamp in the correct manner. \\
Order efficiently lab tests such as U + Es and liver U/S. \\
Can accurately assess IOP. \\
Can accurately record findings. \\
Demonstrates ability to examine the fundus using a 90-D and 20-D Volk lens. \\
Demonstrates an understanding of the anatomy related to normal fundus and can \\
identify landmarks. \\
Demonstrates an understanding of the causes of any deviation from the normal fundus. \\
Can show understanding of normal OCT. \\
Demonstrates awareness of problems requiring medical intervention. \\
Provides verbal explanation of eye condition to patient/carer. \\
Demonstrates the ability to provide the doctor in charge of the Ocular Oncology clinic and the fellow \\
with a clear account of the examination.
\end{tabular}


Table 2 Patients seen by the ophthalmic nurse practitioner during the study period

\begin{tabular}{|c|c|c|c|}
\hline Diagnosis & Tumour location & Anterior margin & Treatment \\
\hline \multirow[t]{2}{*}{ Naevus: 35} & \multirow[t]{2}{*}{ Choroid: 35} & Equator to ora: 15 & \multirow{3}{*}{ Observation } \\
\hline & & Posterior equator: 20 & \\
\hline \multirow[t]{2}{*}{ Uncertain: 3} & \multirow[t]{2}{*}{ Choroid: 3} & Equator to ora: 2 & \\
\hline & & Posterior equator: 1 & Observation \\
\hline Osteoma: 1 & Choroid: 1 & Posterior equator: 1 & Observation \\
\hline Combined hamartoma: 1 & RPE: 1 & Posterior equator: 1 & Observation \\
\hline \multirow[t]{6}{*}{ Melanoma: 16} & \multirow[t]{4}{*}{ Choroid: 13} & Equator to ora: 8 & $\mathrm{Ru}^{106}$ plaque: 5 \\
\hline & & Posterior equator: 4 & Enucleation: 4 \\
\hline & & Iris: 1 & PBT: 3 \\
\hline & & \multirow{3}{*}{ Angle: 3} & Endoresection: 1 \\
\hline & \multirow[t]{2}{*}{ Ciliary body: 3} & & PBT: 2 \\
\hline & & & $\mathrm{Ru}^{106}$ plaque: 1 \\
\hline \multirow[t]{4}{*}{ Patients with monosomy 3: 9} & \multirow[t]{3}{*}{ Choroid: 8} & Posterior equator: 4 & Endoresection: 4 \\
\hline & & Equator to ora: 4 & Enucleation: 3 \\
\hline & & & $\mathrm{Ru}^{106}$ plaque: 1 \\
\hline & Ciliary body: 1 & Angle: 1 & PBT: 1 \\
\hline
\end{tabular}

Abbreviations: PBT, proton beam therapy; $\mathrm{RPE}$, retinal pigmented epithelium; $\mathrm{R}^{106}$, plaque: ruthenium plaque.

(ie, 'suspicious naevus'), which was located in the choroid in all patients. The anterior tumour margin was post-equatorial in 20 patients and pre-equatorial choroid in 15 patients. Three patients attended with lesions of uncertain diagnosis. Two patients with the diagnosis of osteoma and combined hamartoma of the retinal pigmented epithelium, respectively, also attended the nurse-led clinic. The follow-up time from their initial assessment by the consultant ocular oncologist had a median of 36 months (range 6-48 months). The further management consisted of: (a) review at the NOC in 36 patients $(90 \%)$ and (b) review at the ophthalmologist's ocular oncology clinic in 4 patients $(11 \%)$. Three of the patients reviewed at the ophthalmologist's ocular oncology clinic had a diffuse lesion with undefined borders. One patient with an atypical choroidal naevus, who had previously been discharged from the LOOC to a general ophthalmic clinic in our hospital, was recruited into the NOC, only for our nurses to discover that the tumour had grown, also developing clinical features of melanoma (ie, 'orange pigment' and serous retinal detachment (Figures $3 \mathrm{a}$ and $\mathrm{b}$ )).

Anonymised satisfaction questionnaires indicated that patients felt very reassured by the nurse and very satisfied with this new clinic with all the patients scoring the questionnaires as 'five out of five'.

\section{Treated patients}

There were 16 patients under surveillance following treatment of a uveal melanoma, which was confined to choroid in 13 and which involved ciliary body in 3 .
In those patients with a choroidal tumour, the anterior tumour margin was in the post-equatorial choroid in four patients, pre-equatorial in eight, and located in the iris in one patient. In the three patients with a ciliary body melanoma, the anterior tumour margin was located in the angle. The primary treatment in these 16 patients had consisted of ruthenium plaque radiotherapy (6 patients), proton beam treatment (PBT) (5 patients), enucleation (4 patients), and endoresection (1 patient). The follow-up time had a median of 24 months (range 12-84 months). Tumour recurrence was detected by a nurse in one patient. Other ocular morbidity was detected in six patients, consisting of macular oedema in one patient and cataract in five. The further management consisted of: (a) review at the nurse-led clinic in 11 patients and (b) review-back at the ophthalmologist's ocular oncology clinic in 5 patients. These five patients included one patient with conjunctival melanosis at the plaque site, one with macular oedema, and another one with suspicion of tumour recurrence.

Anonymised satisfaction questionnaires, completed by all the patients, highlighted the patients' satisfaction with the extra time they felt they had to discuss their concerns.

\section{High-risk patients}

Nine patients attended the NOC because they had an increased risk of metastatic disease from uveal melanoma. These patients (five male and four female) had a median age of 54 years (range $32-75$ years). The time from primary ocular treatment was 18 months (range 6-18 months). The primary ocular treatment was: 
endoresection (four patients), enucleation (three patients), PBT (two patients), and plaque radiotherapy (one patient). The 5-year risk of metastatic death was estimated to be $10 \%$ in seven patients and $>10 \%$ but $<30 \%$ in two patients. During the period of the study, no metastatic disease was detected. The further management consisted of review at the NOC in all patients.

Anonymised satisfaction questionnaires for this subgroup of patients reinforced the importance of the presence of the trainee health psychologist with six patients requiring such care.

\section{Discussion}

We have established a nurse-led ocular oncology outpatient clinic for the long-term surveillance and counselling of adult patients with an ocular tumour. The success of this service depended on the availability of highly-trained nursing staff, protocols, proformas, patient satisfaction questionnaires, high-quality imaging, and readily available support from photographer, trainee health psychologist, ocular oncologist, and other personnel. Tumour growth, macular oedema, and cataract were identified. Patients expressed high levels of satisfaction with this service.

To our knowledge, a nurse-led ocular oncology clinic has not previously been reported. Until recently, such a clinic would have been inconceivable because of the lethal nature of ocular tumours and the special ophthalmoscopic skills that are required to detect tumour growth or recurrence. The main weakness of this study is that such adverse events may have occurred without being detected by the nurse so that longer follow-up studies and more patients will be required to reveal any failures or shortcomings. Another limitation of this study is that replication at other oncology centres, essential to confirm the generalisation of the findings, might prove difficult to organise. Some ophthalmic oncology centres are staffed entirely by ophthalmologists so that they do not have any trained nurses who can provide unsupervised care.

We anticipated that some patients might object to being reviewed by a nurse and not an ophthalmologist. The nurse was therefore advised to show the patients their own photographs, both current and previous, so that they and any accompanying persons could see for themselves whether or not any change in the tumour had occurred. The responses provided in the anonymous questionnaires indicate that patients were adequately reassured by their follow-up.

Regardless of the patients' views, it is essential for their family doctor, referring ophthalmologist, ocular oncologist, health commissioners, and the nurses themselves to be convinced of the safety of the nurse-led clinic. We therefore took a number of precautions, such as special training and supervision, availability of multidisciplinary support, detailed protocols, proformas, careful patient selection by an ocular oncologist, and continuous audit. The proformas in the casenotes reminded the nurse of all procedures that needed to be taken, as well as enhancing documentation of all findings. This policy of avoiding free text is followed also in the ophthalmologist's ocular oncology clinic for the same reasons. In addition, we ensured that the NOC would meet the same standards as in the ophthalmologist's ocular oncology clinic by providing audio recordings of explanations to patients, sending reports to all carers after each visit, and copying such letters to the patients.

The nurse-led clinic has become possible only because of the recent availability of wide-angle fundus photographs, obtained with the Optos camera. This is because such photographs: (1) greatly facilitate comparison of sequential photographs, without requiring expertise in ophthalmoscopy; (2) enable the nurse to prove to patients whether or not a tumour has changed; (3) enable the nurse to obtain a second opinion from an ophthalmologist, even if the patient is no longer present in the clinic; and (4) provide documentation if any medico-legal problems arise (eg, if a patient alleges that growth or recurrence of a tumour was not detected in a timely manner).

For these reasons, patients were excluded from the nurse-led clinic if their tumour was not adequately photographed.

One might expect that patients would not be satisfied with explanations and reassurances provided by a nurse, preferring to speak to an ophthalmologist. This did not prove to be the case. This was probably because patients tend to speak more freely to a nurse than a doctor because they do not wish to take up too much of the doctor's time, especially in the middle of a busy clinic. Another explanation is that the nurse had previously gained the trust of the patients, having spoken to them in a counselling room at the initial visit and having provided further advice and support during their inpatient stay, as well as after discharge from hospital, by means of proactive telephone calls. Higher levels of patient satisfaction with the nurse care, than with the equivalent doctor-led care, have been reported previously. ${ }^{7}$

Our criteria for systemic screening at NOC were determined by our local general oncologist who was only prepared to accept referrals if the 5-year risk of metastatic death exceeded $50 \%$. This was because he was conducting a formal clinical trial evaluating the role of screening for metastasis with MRI, with limited 
availability of this test at his hospital because of competing demands on this resource. Our selection criteria would change if the general oncologist were to accept patients with a better survival probability. Initially, we were obliged to perform our screening with liver ultrasonography but during the course of this study MRI became available to us at our hospital. We are considering the scope of performing MRI with contrast.

More than one nurse was present at each NOC and this was done so that if one nurse ever becomes indisposed, because of illness or retirement, the clinic can continue without disruption of the service.

Two patients were found to have tumour growth, one of whom had previously been treated. In addition, five patients were found to have conditions such as macular oedema and cataract requiring treatment. All these patients were referred to the ophthalmologist's ocular oncology clinic. Such a referral would probably have taken place even if they had been seen in a general ophthalmology clinic so that the patients were not worse off because they were reviewed by a nurse. Interestingly, the growth of the untreated tumour had been missed in a general ophthalmic clinic and the patient was fortunate that she had been selected randomly to attend our NOC.

The nurse-led model of follow-up has been accepted in other settings, such as in breast and lung cancer, diabetic retinopathy screening, and emergency eye clinics. ${ }^{8-13}$ The role of the nurse-led clinic in an acute care setting has at least a 15-year history in the United Kingdom. ${ }^{12}$

The main benefit of the nurse-led clinic is that it provides patients with specialist expertise and equipments that are not available in general ophthalmic clinics while reducing the workload of the ophthalmologist's ocular oncology clinic. At present, only small numbers of patients are attending the nurseled clinic, but more will be seen at each session as the nurses gain more experience and confidence.

Further studies are needed to identify any shortcomings of the nurse-led clinic and to rectify such problems. Many patients travel long distances so that there may be scope for 'virtual clinics', whereby the nurses review photographs instead of seeing the patients themselves. Such images could be taken by an ophthalmic photographer at the patient's local hospital or by the patient's optometrist without taking up the time of the patient's local ophthalmologist.

In conclusion, we have developed a system for nurse-led surveillance and counselling of adult patients with an ocular tumour. Our initial results are encouraging but further studies are needed to identify any weaknesses and improve efficiency.

\section{Summary}

\section{What was known before}

- Recent improvements in ocular photography have made it easier to detect tumour change over time.

What this study adds

- We have developed the first nurse-led surveillance and counselling of adult patients with an ocular tumour.

\section{Conflict of interest}

The authors declare no conflict of interest.

\section{References}

1 Brandberg Y, Damato B, Kivelä T, Kock E, Seregard S. The EORTC ophthalmic oncology quality of life questionnaire module (EORTC QLQ-OPT30). Development and pre-testing (Phase I-III). Eye 2004; 18(3): 283-289.

2 Cella DF, Tulsky DS, Gray G, Sarafian B, Linn E, Bonomi A et al. The functional assessment of cancer-therapy scale: development and validation of the general measure. J Clin Oncol 1993; 11(3): 570-579.

3 Zigmond AS, Snaith RP. The hospital anxiety and depression scale. Acta Psychiatr Scand 1983; 67(6): 361-370.

4 Undén AL, Orth-Gomér K. Development of a social support instrument for use in population surveys. Soc Sci Med 1989; 29(12): 1387-1392.

5 Folkman S. Personal control and stress and coping processes: a theoretical analysis. J Pers Soc Psychol 1984; 46(4): 839-852.

6 Hoffman BM, Zevon MA, D'Arrigo MC, Cecchini TB. Screening for distress in cancer patients: the NCCN rapid-screening measure. Psychooncology 2004; 13(11): 792-799.

7 Bonsall K, Cheater FM. What is the impact of advanced primary care nursing roles on patients, nurses and their collegues? A literature review. Int J Nurs Stud 2008; 45: 1090-1102.

8 Brown L, Payne S, Royle G. Patient initiated follow up of breast cancer. Psychooncology 2002; 11: 346-355.

9 Grunfeld E, Mant D, Yudkin P, Adewuyi-Dalton R, Cole D, Stewart $\mathrm{J}$ et al. Routine follow up pf breast cancer in primary care: randimised trial. Brit Med J 1996; 313: 665-669.

10 Kirkwood BJ, Coster DJ, Essex RW. Ophthalmic nurse practitioner led diabetic retinopathy screening. Results of a 3-month trial. Eye 2006; 20: 173-177.

11 Kirkwood BJ, Pesudovs K, Loh RS, Coster DJ. Implementation and evaluation of an ophthalmic nurse practitioner emergency eye clinic. Clin Exp Ophthalmol 2005; 33: 593-597.

12 Marsden J. The nurse practitioner role in a United Kingdom ophthalmic accident and emergency department - 10 years of progress. Insight 1999; 24: 45-50.

13 Moore SM, Corner J, Haviland J, Wells M, Salmon E, Charles $\mathrm{N}$ et al. Nurse led follow up and conventional medical follow up in management of patients with lung cancer: randomised trial. Brit Med J 2002; 325: 1-7. 